\title{
Toxina botulínica tipo A y ondas de choque para el tratamiento de dolores crónicos en fascitis plantar
}

\author{
Ana María Fraile Lomo ${ }^{1}$
}

Fecha de recepción: 24 de mayo de 2018 / Fecha de aceptación: 11 de diciembre de 2018

Resumen. La fascitis plantar es un trastorno común del pie que afecta a 1 de cada 10 personas de entre 40 y 60 años, afectando a la actividad diaria en pacientes especialmente deportistas y con sobrepeso. Se han descrito numerosos tratamientos que se llevan a cabo y que en su mayoría ejercen un efecto positivo en la mejora del dolor. En esta revisión se realiza una recopilación de estudios incluidos en meta-análisis sobre la efectividad de la infiltración de toxina botulínica tipo A y ondas de choque extracorpóreas cuando otros tratamientos conservadores no han sido satisfactorios. Ambos tratamientos resultaron ser bastante alentadores mejorando el dolor hasta en un $60 \%$ en la gran mayoría de los pacientes estudiados.

Palabras clave: Fascitis plantar; toxina botulínica tipo A (Botox); terapia de ondas de choque extracorpóreas; metaanálisis; revisión sistemática.

\section{[en] Botulinum toxin type A and shock waves for the treatment of chronic pains in plantar fasciitis}

Abstract. Plantar fasciitis is a common foot disorder that affects 1 in 10 people between 40 and 60 years old, affecting daily activity in especially athletic and overweight patients. Numerous treatments have been described that are carried out and that mostly exert a positive effect on the improvement of pain. In this review, a compilation of studies included in meta-analysis on the effectiveness of the infiltration of botulinum toxin type A and extracorporeal shock waves is performed, when other conservative treatments have not been satisfactory. Both treatments proved to be quite encouraging, improving pain by up to $60 \%$ in the vast majority of patients studied.

Keywords: Plantar fasciitis; botulinum toxin type A (Botox); extracorporeal shock wave therapy (ESWT); metaanalysis; systematic review.

Sumario: 1. Introducción. 2. Metodología. 2.1. Búsqueda estratégica para la identificación de estudios. 2.2. Extracción de datos. 3. Desarrollo. 3.1. Resultados. 3.1.1. Tratamiento con toxina botulínica tipo A para pacientes con fascitis plantar. 3.1.2. Tratamiento con ondas de choque extracorpóreas para fascitis plantar. 3.2. Discusión. 3.3. Conclusiones. 4. Referencias bibliográficas.

Cómo citar: Fraile Lomo, A. M. (2019) Toxina botulínica tipo A y ondas de choque para el tratamiento de dolores crónicos en fascitis plantar, en Revista internacional de ciencias podológicas 13(1), 87-97.

La autora declara no tener ningún tipo de interés económico o comercial.

\footnotetext{
Grado en Podología Ana María Fraile Lomo

Fremap Móstoles - Calle E número 5 (Pol. Ind. Arroyomolinos). Móstoles 28938 (Madrid) anamaria.fl@hotmail.com
} 


\section{Introducción}

La fascitis plantar es un trastorno común del pie que se manifiesta con dolor localizado en la inserción de la fascia plantar cerca del tubérculo medial del calcáneo, siendo el peor momento, en el primer paso de la mañana. Las personas obesas, las mujeres, y los atletas que participan en actividades de carrera frecuentemente tienen un mayor riesgo de desarrollar esta patología.(1)

Se estima que aproximadamente 1 de cada 10 personas experimenta este dolor de talón en algún momento. Aunque la fascitis plantar ocurre en todas las edades, el mayor riesgo de aparición es de 40 a 60 años de edad, sin sesgo de sexo significativo (2).

La etiología de la fascitis plantar es probablemente multifactorial y muy poco conocida.(3). Sin embargo, la carga de peso en el trabajo, un pie con tendencia a la pronación, la discrepancia de la longitud de la extremidad, los espolones óseos y la obesidad son considerados factores de riesgo para favorecer el desarrollo de una fascitis plantar.(4)

El dolor plantar crónico es un término que generalmente se utiliza para describir un rango de condiciones indiferenciadas afectando al talón plantar. El dolor crónico debajo del talón es la clínica típicamente descrita que empeora a la bipedestación después de períodos prolongados de descanso.(5)

Las opciones de tratamiento para aliviar los síntomas de la fascitis plantar incluyen entre otros, la modificación de la actividad, estiramientos de la fascia plantar, masajes con hielo, férulas nocturnas, antiinflamatorios no esteroideos combinados con otras modalidades de tratamiento, inyecciones de esteroides locales, terapia de ondas de choque extracorpóreas y cirugía.(6)Sin embargo, en los últimos años la toxina botulínica tipo A se ha utilizado para el tratamiento crónico muscular y el dolor neuropático.(7)

La toxina botulínica tipo A (TbA) es una neurotoxina que actúa inhibiendo la liberación del neurotransmisor, la acetilcolina, en uniones neuromusculares, reduciendo así las contracciones musculares.(8)La infiltración se realiza bajo guía ecográfica que facilita la precisión y aumenta los efectos terapéuticos(9), pudiendo relajar la tensión de la fascia plantar y el complejo muscular gastrosóleo (10).

Sin embargo, a pesar del potencial terapéutico de la toxina botulínica tipo A para aliviar espasmos musculares dolorosos, su eficacia en otros dolores musculo-esqueléticos no están bien establecidas, incluso su uso en el dolor miofascial. (11)

En comparativa, una opción como tratamiento alternativo para la fascitis plantar son las terapias de ondas de choque extracorpóreas (ESWT) que se han utilizado ampliamente durante décadas debido a su naturaleza no invasiva, el tiempo de recuperación rápido y la comodidad para la vida diaria de los pacientes(12)sin la necesidad de reducir la carga de peso o la inmovilización. La justificación para el usode ondas de choque para estas afecciones se basa en la estimulación de la cicatrización de tejidos blandos mediante hiperemia local, neovascularización, reducción de la calcificación, inhibición de los receptores del dolor y denervación para lograr el alivio del dolor y la curación persistente de los procesos crónicos (13).

En este estudio reflejaremos la búsqueda de los tratamientos utilizados hasta el momento enfocando nuestra atención en las terapias de ondas de choque extracorpóreas que se han utilizado con una frecuencia cada vez mayor para tratar diversas lesiones muculoesqueléticas, como epicondilitis lateral (14) 2004; y la aplicación de toxina botulínica tipo A, todavía en estudio, con el fin de valorar resultados y definir el tratamiento más eficaz en pacientes con fascitis plantar crónica donde otros tratamientos conservadores no han sido definitivos.

\section{Metodología}

\subsection{Búsqueda estratégica para la identifi- cación de estudios:}

La pregunta clínica para esta revisión sistemática se realizó utilizando el formato PICO (15) siendo de la siguiente manera: ¿En la fascitis plantar crónica, la infiltracióncon toxina botulínica tipo A y las terapias de ondas de choque extracorpóreas serían efectivas cuando otros tratamientos no han dado los resultados esperados en pacientes que demandan la retoma de la actividad diaria o deportiva?

$\mathbf{P}$ pacientes (grupo de edad, estadio de la fascitis plantar crónica) 
I intervención (tipo de tratamiento: infiltración de toxina botulínica tipo $\mathrm{A}(\mathrm{tbA})$ y ondas de choque extracorpóreas)

C comparativa (infiltración de tbA y ondas de choque extracorpóreas versus placebo según dosis).

O "outcomes" o resultados ((pacientes con fascitis plantar con dolor mantenido según la escala visual analógica (EVA) al menos durante los últimos 3 meses))

La búsqueda se realizó en primer lugar por términos de enfermedad, en este caso "plantar fasciitis", encontrándose gran número de artículos; sin embargo, la búsqueda de tratamiento con $\mathrm{TbA}$ en fascitis plantar resultó ser bastante escasa, ya que los estudios publicados con este tipo de infiltraciones están relacionados con otro tipo de patologías neuromusculares con sintomatología asociada. En cuanto a los artículos sobre el tratamiento con ondas de choque extracorpóreas los resultados fueron agradecidos en la comparación con los niveles de energía aplicada.
Estas búsquedas se realizaron a través de las bases de datos de Pubmed, Medline, Cochrane library y PEDRO para identificar estudios de ensayos tipo meta-análisis (MA) y revisiones sistemáticas (RS) utilizando palabras clave en inglés para lo cual se encontraron diversos resultados (tabla 1 ).

Acotamos nuestra elección de estudios a aquellos que relacionaban la fascitis plantar con el tratamiento comparativo:

- "Botulinum toxin type A and plantar fasciitis"

- "Extracorporeal shock wave therapy and plantar fasciitis"

- "Extracorporeal shock wave therapy and botulinum toxin type A"

Tras ser eliminados los estudios duplicados se revisó la lista de referencias de los artículos encontrados y se incluyeron aquellos artículos que cumplen los criterios de inclusión (tabla 2).

Tabla 1. Resultados de búsquedas según grupos de interés

\begin{tabular}{|l|c|c|c|c|}
\hline \multicolumn{1}{|c|}{$\begin{array}{c}\text { Términos de } \\
\text { búsqueda }\end{array}$} & Pub Med & Medline & PEDRO & $\begin{array}{c}\text { Cochrane } \\
\text { library }\end{array}$ \\
\hline Plantar fasciitis & 1287 & 869 & 124 & 23 \\
\hline $\begin{array}{l}\text { Botulinum toxin type } \\
\text { A and plantar fasciitis }\end{array}$ & 30 & 18 & 0 & 1 \\
\hline $\begin{array}{l}\text { Botulinum toxin type } \\
\text { A and pain }\end{array}$ & 2394 & 1008 & 13 & 32 \\
\hline $\begin{array}{l}\text { Extracorporeal shock } \\
\text { wave therapy }\end{array}$ & 4766 & 1689 & 129 & 23 \\
\hline $\begin{array}{l}\text { Extracorporeal shock } \\
\text { wave therapy and } \\
\text { plantar fasciitis }\end{array}$ & 155 & 120 & 28 & 5 \\
\hline $\begin{array}{l}\text { Extracorporeal shock } \\
\text { wave therapy and } \\
\text { botulinum toxin type A }\end{array}$ & 4 & 16 & 2 & 1 \\
\hline
\end{tabular}


Tabla 2. Criterios de inclusión y exclusión de artículos

\begin{tabular}{|c|c|}
\hline Criterios de inclusión & Criterios de exclusión \\
\hline $\begin{array}{l}\text { Tipo de estudio: } \\
\quad \text { Meta-análisis } \\
\quad \text { Revisiones sistemáticas } \\
\quad \text { ECA } \\
\text { Participantes: } \\
\text { - Adultos mayores de } 18 \text { años } \\
\text { - Fascitis plantar crónica (más de } 6 \text { meses de evo- } \\
\text { lución donde el tratamiento conservador no ha } \\
\text { sido efectivo) } \\
\text { Tipo de intervención: } \\
\text { Terapia de ondas de choque de alta energía } \\
\text { Infiltración de toxina botulínica tipo A } \\
\text { Comparación: } \\
\text { Placebo } \\
\text { Outcomes: } \\
\text { Dolor según Escala Visual Analógica (EVA) del } \\
0 \text {-10 }\end{array}$ & $\begin{array}{l}\text { Tipo de estudio: } \\
\text { - Estudios de cohorte y casos con control } \\
\text { Artículos no publicados en inglés } \\
\text { Artículos que no ofrecían tratamiento comparativo } \\
\text { con placebo } \\
\text { Artículos publicados hace más de } 10 \text { años }\end{array}$ \\
\hline
\end{tabular}

Se obtuvieron ciento ochenta y nueve se encontraron cinco textos significativos, y artículos; de estos, ciento treinta y tres fueron se incluyeron tres publicaciones dentro de la excluidos según los criterios de inclusión / revisión (Figura 1).

exclusión. Tras la revisión del texto completo,

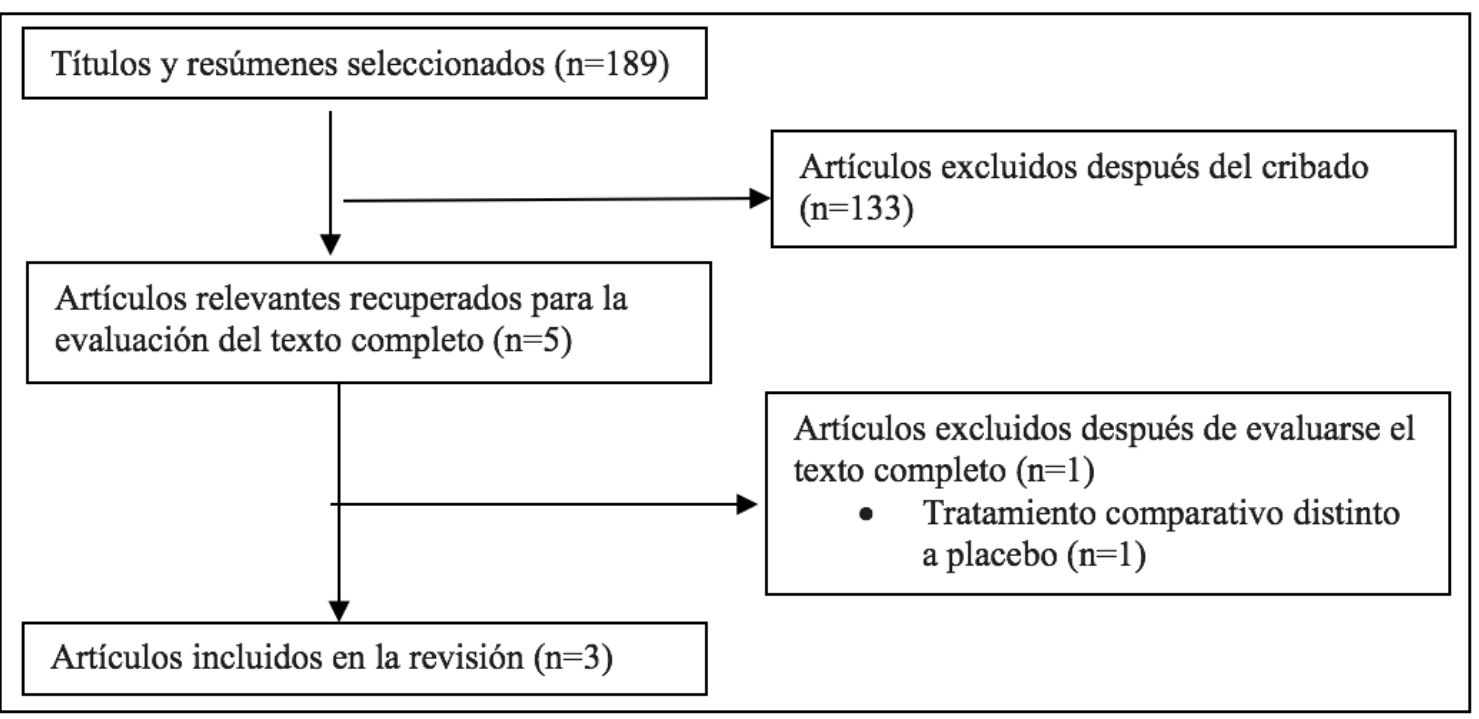

Figura1. Repaso de literatura 


\subsection{Extracción de datos:}

Todos los artículos encontrados en los MA y RS escogidos se clasificaron según los niveles de evidencia utilizando los criterios establecidos por el Oxford Centre for Evidence Medicina(16), niveles de calidad de evidencia científica (AATM) (17) que se encuentran entre el I y el III: Meta-análisis de ECA, ECA de muestra grande y ECA de muestra pequeña.

\section{Metodología}

\subsection{Resultados}

De los meta-análisis que fueron escogidos según los criterios de inclusión, se analizaron los estudios de ensayos clínicos aleatorizados (ECA) y se escogieron aquellos que cumplían las características de estudio en cuanto al tipo de intervención (tratamiento) y el grupo control (placebo).

Se realizó un resumen de los artículos encontrados elaborando una tabla con las características más importantes para el tratamiento con TbA (tabla 3) y para el tratamiento con ondas de choque extracorpóreas (tabla 4).
Todos los resultados fueron evaluados según la Escala Visual Analógica (EVA) en la fase inicial de los estudios y al finalizar el tratamiento, donde 1 es un dolor leve y 10 un dolor insoportable.

\subsubsection{Tratamiento con toxina botulínica tipo A para pacientes con fascitis plantar}

Se evaluó solo un estudio de cada meta-análisis incluido, al ser los únicos que analizaban específicamente el efecto de la infiltración con TbA en la fascitis plantar. Ambos escogían un número " $n$ " de personas entre los $20 \mathrm{y}$ los 65 años de edad en la que predominaban el número de mujeres frente a hombres. (Tabla 3 )

Uno de ellos (20), incluyó aquellos casos que padecían dolor localizado en tuberosidad medial de calcáneo con al menos 6 meses de evolución y se trató con mayor dosis de TbA tipo Botox.

Por el contrario, el estudio comparativo (21), analizó los casos aleatorizados que padecían la enfermedad con tan solo 3 meses y se llevó un seguimiento tras el tratamiento más prolongado respecto al anterior.

La mayoría de estos pacientes experimentaron dolor durante al menos 3 meses con poca o ninguna mejoría y sin respuesta favorable a otros tratamientos orales frente al dolor.

Tabla 3. Características de los estudios incluidos en la revisión de infiltración con toxina botulínica para pacientes con fascitis plantar.

\begin{tabular}{|c|c|c|c|c|c|c|c|c|c|}
\hline $\begin{array}{c}\text { Autor y } \\
\text { Año }\end{array}$ & $\begin{array}{c}\text { País } \\
\text { y tipo } \\
\text { población }\end{array}$ & $\begin{array}{c}\text { Tipo } \\
\text { estudio }\end{array}$ & $\begin{array}{c}\text { Edad } \\
\text { media }\end{array}$ & $\begin{array}{c}\text { Punto } \\
\text { anatómico de } \\
\text { dolor }\end{array}$ & $\begin{array}{c}\text { Duración } \\
\text { del dolor }\end{array}$ & Intervención & $\begin{array}{c}\text { Grupo } \\
\text { control } \\
\text { (pasivo) }\end{array}$ & $\begin{array}{c}\text { Semanas de } \\
\text { seguimiento }\end{array}$ & $\begin{array}{c}\text { Nivel de } \\
\text { evidencia } \\
\text { de } \\
\text { estudios }\end{array}$ \\
\hline $\begin{array}{c}\text { Babcock } \\
\text { et al. } \\
(34)\end{array}$ & $\begin{array}{c}\text { USA } \\
\mathrm{n}=27 \\
>\text { mujere } \\
(2005)\end{array}$ & $\begin{array}{c}\text { ECA } \\
\text { prospectivo } \\
\text { paralelo, } \\
\text { doble ciego }\end{array}$ & $44 \mathrm{a}$ & $\begin{array}{c}\text { Tuberosidad } \\
\text { medial de } \\
\text { calcáneo }\end{array}$ & $6 \mathrm{~m}$ & $70 \mathrm{U}$ (Botox) & $\begin{array}{c}0,7 \mathrm{ml} \\
\mathrm{SS} \text { o } \\
\text { placebo }\end{array}$ & $8 \mathrm{~s}$ & III \\
\hline $\begin{array}{c}\text { Huang y } \\
\text { co. (35) } \\
(2010)\end{array}$ & $\begin{array}{c}\text { China } \\
\mathrm{n}=50\end{array}$ & $\begin{array}{c}\text { ECA doble } \\
\text { ciego }\end{array}$ & $54,4 \mathrm{a}$ meres & $\begin{array}{c}1-2 \text { cm distal } \\
\text { tuberosidad } \\
\text { medial de } \\
\text { calcáneo }\end{array}$ & $3 \mathrm{~m}$ & $50 \mathrm{U}$ (Botox) & $1 \mathrm{ml} \mathrm{SS}$ & $12 \mathrm{~s}$ & II \\
\hline
\end{tabular}

En cuanto a la realización de la infiltración de TbA, dos de los estudios encontrados la realizan de manera diferente. Huang y co. utiliza la prueba de imagen mediante ECO para guiarse y ser más específico en la inyección de toxina botulínica en la zona de inserción de la fascia plantar. Babcock et al. realiza dos tipos de infiltraciones, una en la zona del arco interno de la fascia y la otra proximal a la inserción, y utiliza mayor dosis en la inyección (70U). El grosor de la fascia plantar también pareció ser una variable a tener en cuenta en el resultado del 
tratamiento.La infiltración con toxina botulínica tipo A demostró ser eficaz en la fascitis plantar en todos los estudios analizados (tabla 4). Sin embargo, los estudios con TbA para pacientes con fascitis plantar están muy limitados debido su escasa utilización para esta patología.

Tabla 4. Reducción del dolor según puntuación de la escala VAS tras el tratamiento con tbA y grupo control evaluados desde el inicio y hasta los tres meses posteriores.

\begin{tabular}{|c|c|c|c|}
\hline \multirow{2}{*}{ Estudio } & Seguimiento & $\begin{array}{c}\text { TbA } \\
\text { (\%reducción dolor) }\end{array}$ & $\begin{array}{c}\text { Placebo } \\
\text { (\%reducción dolor) }\end{array}$ \\
\hline \multirow{3}{*}{ Babcock et al } & $\mathrm{A}$ inicio & 5.1 & 4.9 \\
\cline { 2 - 4 } & $\mathrm{A}$ las 3 semanas & $2.7(-57 \%)$ & $4.7(-4,1 \%)$ \\
\cline { 2 - 4 } & $\mathrm{A}$ los 3 meses & $1.6(-68,7 \%)$ & $4.4(-10,3 \%)$ \\
\hline \multirow{3}{*}{ Huang et al. } & $\mathrm{Al}$ inicio & 5.9 & 5.4 \\
\cline { 2 - 4 } & $\mathrm{A}$ las 3 semanas & $3.4(-42,4 \%)$ & $5.1(-5,6 \%)$ \\
\cline { 2 - 4 } & $\mathrm{A}$ los 3 meses & $2.0(-66,1 \%)$ & $5.2(-3,8 \%)$ \\
\hline
\end{tabular}

\subsubsection{Tratamiento con ondas de choque extracorpóreas para fascitis plantar}

El estudio de este tema se realizó a través de tres artículos escogidos del meta-análisis de Jiale Sun et al. (18) que comparan el tratamiento con la misma dosis de frecuencia de onda (2000 impulsos) con dosis placebo a través de piezas que realizan un bloqueo en la zona anatómica impidiendo la transmisión de impulsos desde el aplicador hasta la piel.

Para comparar el efecto de las ondas de choque con el efecto placebo para la fascitis plantar, los autores reparten un número $\mathrm{n}$ de pacientes por igual en el grupo de tratamiento y en el grupo control.
Todos los resultados fueron evaluados a las 3 sesiones de tratamiento salvo el estudio de Ibrahim et al. (19) que lo realizó en 2 sesiones. (Tabla 5). Cada uno realizó un seguimiento diferente, el estudio de Gollwitzer et al. sólo evaluó a los casos pasadas las 12 semanas desde el comienzo del tratamiento y fue suficiente para indicar que las ondas de choque focales son claramente más efectivas que el tratamiento con placebo para fascitis plantar.

En todos ellos se experimentó una mejoría de hasta un $60 \%$ en la escala analógica de EVA del dolor desde el inicio del tratamiento con ondas de choque extracorpóreas, dando resultados positivos casi en el $100 \%$ de los pacientes en el estudio de Ibrahim et al. (Tabla 6). 


\begin{tabular}{|c|c|c|c|}
\hline 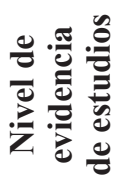 & 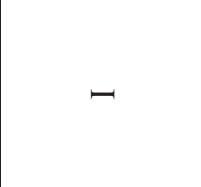 & $\neg$ & - \\
\hline 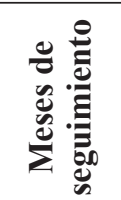 & $\underset{m}{\Xi}$ & ઘี & $\underset{m}{\Xi}$ \\
\hline 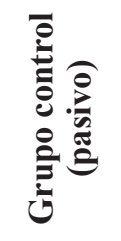 & 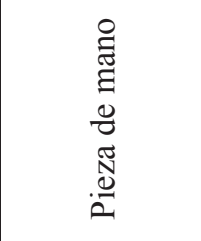 & 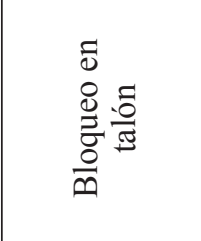 & 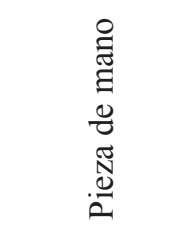 \\
\hline & 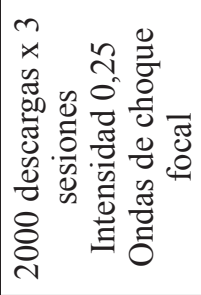 & 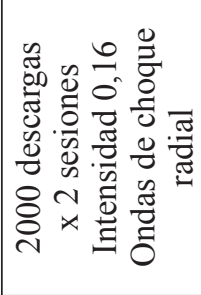 & 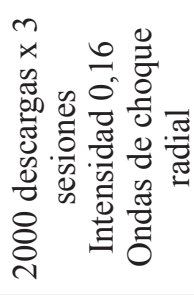 \\
\hline 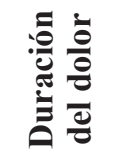 & $\begin{array}{l}\Xi \\
\vdots \\
\wedge\end{array}$ & $\begin{array}{l}\Xi \\
\swarrow\end{array}$ & $\begin{array}{l}\Xi \\
\underset{\wedge}{\sim}\end{array}$ \\
\hline 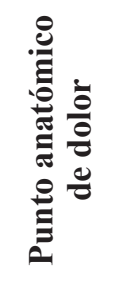 & 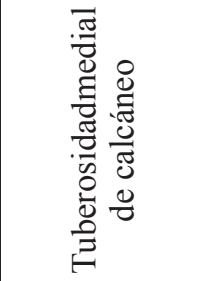 & 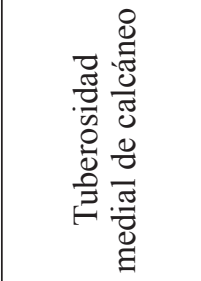 & 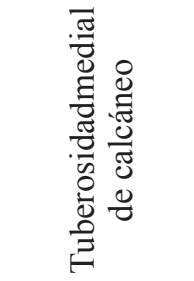 \\
\hline 疍 & $\underset{\sigma}{\stackrel{\sigma}{q}}$ & $\begin{array}{l}\tilde{r} \\
\tilde{n}\end{array}$ & $\begin{array}{l}\tilde{\sigma} \\
\tilde{n}\end{array}$ \\
\hline 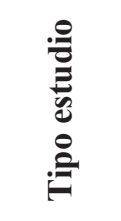 & 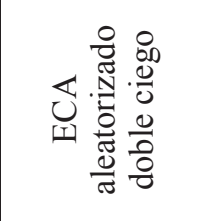 & 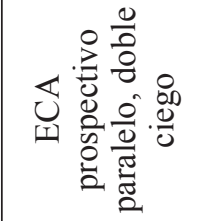 & 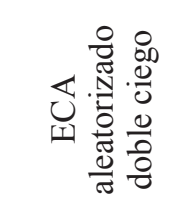 \\
\hline 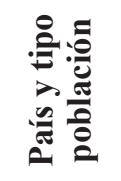 & 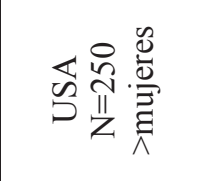 & 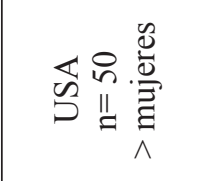 & 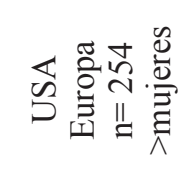 \\
\hline 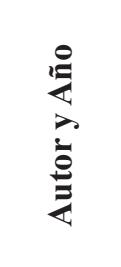 & 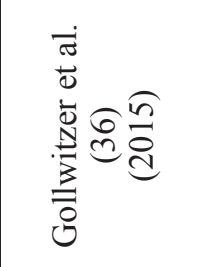 & 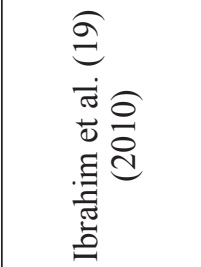 & 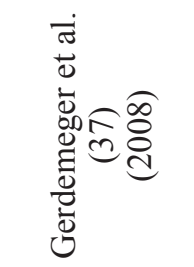 \\
\hline
\end{tabular}


Tabla 6. Resultados y tasas de éxito tras el tratamiento de ondas de choque y grupo placebo

\begin{tabular}{|c|c|c|c|}
\hline Estudios & Grupo & $\begin{array}{c}\text { Tasa de éxito/ tasa } \\
\text { de mejora }\end{array}$ & $\begin{array}{l}\text { Reducción del } \\
\text { dolor }\end{array}$ \\
\hline \multirow{2}{*}{$\begin{array}{l}\text { Gollwitzer et al, } \\
2015\end{array}$} & ESWT & $68 / 125$ & 12 semanas: $-69,2 \%$ \\
\hline & Placebo & $45 / 21$ & 12 semanas: $-34,5 \%$ \\
\hline \multirow{2}{*}{$\begin{array}{l}\text { Ibrahim et al, } \\
2010\end{array}$} & ESWT & $25 / 25$ & $\begin{array}{c}4 \text { meses: }-92,5 \% \\
12 \text { semanas: }-87,3 \% \\
24 \text { semanas: }-93,9 \%\end{array}$ \\
\hline & Placebo & $4 / 25$ & $\begin{array}{c}4 \text { meses: }-15,2 \% \\
12 \text { semanas: }-13,5 \% \\
24 \text { semanas: }-17 \%\end{array}$ \\
\hline \multirow{2}{*}{$\begin{array}{l}\text { Gerdesmeyer et al, } \\
2008\end{array}$} & ESWT & $75 / 125$ & $\begin{array}{c}12 \text { semanas: }-72,1 \% \\
12 \text { meses: }-84.8 \%\end{array}$ \\
\hline & Placebo & $49 / 118$ & $\begin{array}{c}12 \text { semanas: }-44,7 \% \\
12 \text { meses: }-43,2 \%\end{array}$ \\
\hline
\end{tabular}

\subsection{Discusión}

Esta revisión nos ha demostrado que todavía hay poca evidencia científica que aclare ciertamente el tratamiento más eficaz contra el dolor crónico en la tuberosidad medial de calcáneo y el recorrido de la fascia plantar sin que ello impida la realización de la actividad diaria y el ejercicio físico. Este estudio ha servido para realizar una recopilación de los datos más certeros encontrados en las bases de datos y que nos aportan la información necesaria para desarrollar la conclusión.

Hasta la fecha, existen varios tratamientos para la fascitis plantar que se pueden dividir en tratamientos no invasivos, como la fisioterapia (20), ortesis (21), fármacos antiinflamatorios no esteroideos orales (AINE) (22), ultrasonidos (23)y ondas de choque extracorpóreas (18); y tratamientos invasivos, como las infiltraciones (26) y la cirugía (27).

Como última novedad se está usando la inyección de corticosteroides (24), la inyección de toxina botulínica (25), y la inyección de plasma rico en plaquetas (PRP). Este último es ampliamente utilizado en el mundo de la deportiva ya que acelera el periodo de curación favoreciendo factores de crecimiento plaquetario (26).

En cuanto a la infiltración de corticosteroides, hay gran variedad de estudios comparativos que demuestran mejoría en pacientes con procesos agudos de inflamación. En el estudio de Babcock y co. se compara la inyección local de corticoides de $2 \mathrm{ml}$. betametasona, $0,5 \mathrm{ml}$. de mepivacaína y suero salino dando resultados muy positivos.

En un estudio realizado por Diaz-Llopis y co. se compara el tratamiento de las inyecciones de TbA y corticoides, llegando a la conclusión, de que tanto uno como otro tratamiento son efectivos frente a la fascitis plantar recalcitante e incluso podría decirse que se obtuvo mejores resultados en la EVA del grupo tratado con toxina botulínica tipo A (24).

Los efectos analgésicos de la TbA se deben en parte a la reducción del espasmo muscular por inhibición de la liberación de la acetil-colina y por otra a la disminución de la inflamación neurogénica ya que inhibe la liberación de varios neurotransmisores no colinérgicos (28), tales como la sustancia $\mathrm{P}$, Péptido relacionado con el gen de Calcitonina (PRGC), Glutamato y bloqueando las vías autonómicas (29).

Los efectos adversos que pueden presentarse son leves o moderados, siempre transitorios y bien tolerados y no requieren de un tratamiento específico. Las reacciones más comunes que se producen son inflamación local con leves molestias en los músculos inyectados, además de disfagia y disfonía, boca seca, mareo, debilidad mayor a la esperada en los músculos tratados y difusión sistémica provocando el flu-like symptoms (síndrome pseudo-gripal). De forma rara pueden ocurrir efectos adversos serios, como dificultad o insuficiencia respiratoria 
por parálisis de los músculos respiratorios requiriendo incluso el uso de ventilación mecánica durante un período (30). Se ha de ser cauto en el uso de una dosis segura con buen efecto terapéutico, para lograr una baja incidencia de eventos colaterales y con efectos sistémicos prácticamente ausentes.

Sin embargo, la mayoría de los estudios encontrados sobre el tratamiento con inyección de toxina botulínica tipo A lo relacionan a dolores miofasciales, brazo de tenista (epicondilitis), latigazo cervical y dolores de hombro. Son muy pocos los que definen este tratamiento este con el dolor crónico de la fascitis plantar.

Las ondas de choque (ESWT) son utilizadas ampliamente como primera opción cuando otros tratamientos conservadores no tienen los resultados esperados. Sin embargo, tienen muchos posibles efectos secundarios como hematomas, hinchazón, dolor o entumecimiento. En los estudios incluidos no se encontraron efectos adversos graves, aunque algunos pacientes describieron sensación de incomodidad, dolor, hinchazón y hematoma durante o después del tratamiento relacionado con la intensidad de carga. En general, se trata de una indicación terapéutica segura y efectiva (31).

Existen dos tipos de ondas de choque extracorpóreas que se han utilizado en los estudios: ondas de choque radiales (RSWT) y las ondas de choque focales (FSWT). A pesar de que todos los estudios encontrados sobre este tratamiento para fascitis plantar reconocen ser efectivos terapéuticamente ambos tipos, la realidad es que existen diferencias a tener en cuenta. Las RSWT se usan principalmente para casos de tendinitis sobre tejidos más superficiales y sus ondas de choque son propagadas de forma divergente, mientras que las FSWT concuerdan en un punto en concreto propagándose de forma lineal y actuando en tejidos más profundos o puntos gatillos, lo que conlleva que esta última sea una técnica más dolorosa (32).

A diferencia de la cirugía y las infiltraciones, las terapias de ondas de choque extracorpóreas son un tratamiento no invasivo, bien tolerado y relativamente de bajo coste con el que los pacientes vuelven a la vida diaria dentro un corto periodo de tiempo (33).

Todos los estudios que utilizaron el tratamiento con ondas de choque extracorpóreas mostraron una adecuada información en la reducción en la escala EVA del dolor frente al comparativo del grupo control con placebo, especialmente el estudio de Ibrahim et al. que muestra unos resultados altamente positivos en todos los pacientes estudiados.

\subsection{Conclusión}

Se puede concluir que:

- Los tratamientos con placebo tienen una eficacia prácticamente nula frente a las ondas de choque o la toxina botulínica tipo A.

- Las ondas de choque son más efectivas durante los primeros tres meses desde la primera sesión que la infiltración con TbA.

- La infiltración de TbA y la ESWT tienen el mismo efecto terapéutico tras los meses iniciales del tratamiento.

- Las ondas de choque resultan un tratamiento no invasivo frente a la TbA y por lo tanto evitan posibles efectos adversos y mejor calidad cualitativa para el paciente.

\section{Referencias bibliográficas}

1. Tu P. Heel Pain: Diagnosis and Management. Am Fam Physician. 2018 Jan 15;97(2):86-93.

2. Taunton JE, Ryan MB, Clement DB, McKenzie DC, Lloyd-Smith DR, Zumbo BD. A retrospective case-control analysis of 2002 running injuries. Br J Sports Med. 2002 Apr;36(2):95-101.

3. Wearing SC, Smeathers JE, Urry SR, Hennig EM, Hills AP. The pathomechanics of plantar fasciitis. Sports Med. 2006 ;36(7):585-611.

4. Thomas JL, Christensen JC, Kravitz SR, Mendicino RW, Schuberth JM, Vanore J V., et al. The Diagnosis and Treatment of Heel Pain: A Clinical Practice Guideline-Revision 2010. J Foot Ankle Surg. 2010 May;49(3):S1-19.

5. Buchbinder R. Clinical practice. Plantar fasciitis. N Engl J Med [Internet]. 2004 May 20;350(21):215966.

6. Lim AT, How CH, Tan B. Management of plantar fasciitis in the outpatient setting. Singapore Med J. 2016 Apr;57(4):168-70; quiz 171. 
7. Luvisetto S, Marinelli S, Cobianchi S, Pavone F. Anti-allodynic efficacy of botulinum neurotoxin A in a model of neuropathic pain. Neuroscience. 2007 Mar 2;145(1):1-4.

8. Borodic GE, Acquadro M, Johnson EA. Botulinum toxin therapy for pain and inflammatory disorders: mechanisms and therapeutic effects. Expert Opin Investig Drugs. 2001 Aug;10(8):1531-44.

9. Tsai W-C, Hsu C-C, Chen CPC, Chen MJL, Yu T-Y, Chen Y-J. Plantar fasciitis treated with local steroid injection: comparison between sonographic and palpation guidance. J Clin Ultrasound. 2006 Jan;34(1):12-6.

10. Elizondo-Rodriguez J, Araujo-Lopez Y, Moreno-Gonzalez JA, Cardenas-Estrada E, Mendoza-Lemus $\mathrm{O}$, Acosta-Olivo C. A comparison of botulinum toxin A and intralesional steroids for the treatment of plantar fasciitis: A randomized, double-blinded study. Foot Ankle Int. 2013;34(1).

11. Lew MF. Review of the FDA-approved uses of botulinum toxins, including data suggesting efficacy in pain reduction. Clin J Pain;18(6 Suppl):S142-6.

12. Theodore GH, Buch M, Amendola A, Bachmann C, Fleming LL, Zingas C. Extracorporeal shock wave therapy for the treatment of plantar fasciitis. Foot ankle Int. 2004 May 28;25(5):290-7.

13. Rompe JD, Decking J, Schoellner C, Nafe B. Shock wave application for chronic plantar fasciitis in running athletes. A prospective, randomized, placebo-controlled trial. Am J Sports Med. 2003 Mar 30;31(2):268-75.

14. Buchbinder R, Green S, Youd JM, Assendelft WJ, Barnsley L, Smidt N. Shock wave therapy for lateral elbow pain. Cochrane Database Syst Rev. 2005 Oct 19;(4):CD003524.

15. Huang X, Lin J, Demner-Fushman D. Evaluation of PICO as a knowledge representation for clinical questions. AMIA . Annu Symp proceedings AMIA Symp. 2006;359-63.

16. Howick J, Chalmers I, Glasziou P, Greenhalgh T, Heneghan C, Liberati A, et al. The Oxford 2011 Levels of Evidence. Oxford Centre Evidence-Based Medicine. Vol. 1, Group. 2011.

17. Jovell AJ, Navarro-Rubio MD. Evaluación de la evidencia científica. Med Clin. 1995;105:740-3.

18. Sun J, Gao F, Wang Y, Sun W, Jiang B, Li Z. Extracorporeal shock wave therapy is effective in treating chronic plantar fasciitis: A meta-analysis of RCTs. Medicine (Baltimore). 2017 Apr;96(15):e6621.

19. Ibrahim MI, Donatelli R a, Schmitz C, Hellman M a, Buxbaum F. Chronic plantar fasciitis treated with two sessions of radial extracorporeal shock wave therapy. Foot ankle Int / Am Orthop Foot Ankle Soc [and] Swiss Foot Ankle Soc. 2010;31(5):391-7.

20. Cinar E, Saxena S, Uygur F. Combination Therapy Versus Exercise and Orthotic Support in the Management of Pain in Plantar Fasciitis: A Randomized Controlled Trial. Foot Ankle Int. 2018;107110071774759.

21. Struijs PAA, Smidt N, Arola H, Van Dijk CN, Buchbinder R, Assendelft WJJ. Orthotic devices for tennis elbow: A systematic review. Br J Gen Pract. 2001;51(472):924-9.

22. Donley BG, Moore T, Sferra J, Gozdanovic J, Smith R. The Efficacy of Oral Nonsteroidal AntiInflammatory Medication (NSAID) in the Treatment of Plantar Fasciitis: A Randomized, Prospective, Placebo-Controlled Study. Foot Ankle Int. 2007;28(1):20-3.

23. Konjen N, Napnark T, Janchai S. A comparison of the effectiveness of radial extracorporeal shock wave therapy and ultrasound therapy in the treatment of chronic plantar fasciitis: a randomized controlled trial. J Med Assoc Thail. 2015;98 Suppl 1:S49-56.

24. Diaz-Llopis I V., Rodriguez-Ruiz CM, Mulet-Perry S, Mondejar-Gomez FJ, Climent-Barbera JM, Cholbi-LLobel F. Randomized controlled study of the efficacy of the injection of botulinum toxin type A versus corticosteroids in chronic plantar fasciitis: results at one and six months. Clin Rehabil. 2012;26(December):594-606.

25. Zhang T, Adatia A, Zarin W, Moitri M, Vijenthira A, Chu R, et al. The efficacy of botulinum toxin type A in managing chronic musculoskeletal pain: A systematic review and meta analysis. Inflammopharmacology. 2011;19(1):21-34.

26. Mahindra P, Yamin M, Selhi HS, Singla S, Soni A. Chronic Plantar Fasciitis: Effect of Platelet-Rich Plasma, Corticosteroid, and Placebo. Orthopedics. 2016;39(2):e285-9.

27. Ghandour T, Abdelrahman AA, Ghandour A. Evaluation and results of modified deep fascial endoscopic plantar fasciotomy. Eur Orthop Traumatol. 2015;6(3):185-8.

28. Ranoux D, Attal N, Morain F, Bouhassira D. Botulinum toxin type A induces direct analgesic effects in chronic neuropathic pain. Ann Neurol. 2008;64(3):274-83.

29. Klein AW. The therapeutic potential of botulinum toxin. Dermatol Surg. 2004;30(3):452-5.

30. Jeynes LC, Gauci CA. Evidence for the use of botulinum toxin in the chronic pain setting - A review of the literature. Pain Pract. 2008;8(4):269-76.

31. Sun J, Gao F, Wang Y, Sun W, Jiang B, Li Z. Extracorporeal shock wave therapy is effective in treating chronic plantar fasciitis. Medicine (Baltimore). 2017;96(15):e6621.

32. Krol P, Franek A, Durmala J, Blaszczak E, Ficek K, Krol B, et al. Focused and Radial Shock Wave Therapy in the Treatment of Tennis Elbow: A Pilot Randomised Controlled Study. J Hum Kinet. 2015;47(1):127-35. 
33. Zhiyun L, Tao J, Zengwu S. Meta-analysis of high-energy extracorporeal shock wave therapy in recalcitrant plantar fasciitis. Swiss Med Wkly. 2013;143.

34. Babcock MS, Foster L, Pasquina P, Jabbari B. Treatment of pain attributed to plantar fasciitis with botulinum toxin a: a short-term, randomized, placebo-controlled, double-blind study. Am J Phys Med Rehabil. 2005;84(9):649-54.

35. Huang YC, Wei SH, Wang HK, Lieu FK. Ultrasonographic guided botulinum toxin type a for plantar fasciitis: An outcome-based investigation for treating pain and gait changes. J Rehabil Med. 2010;42(2):136-40.

36. Gollwitzer H, Saxena A, DiDomenico LA, Galli L, Bouche RT, Caminear DS, et al. Clinically relevant effectiveness of focused extracorporeal shock wave therapy in the treatment of chronic plantar fasciitis: a randomized, controlled multicenter study. J Bone Jt Surg. 2015;97(9):701-8.

37. Gerdesmeyer L, Frey C, Vester J, Maier M, Lowell W, Weil L, et al. Radial Extracorporeal Shock Wave Therapy is Safe and Effective in the Treatment of Chronic Recalcitrant Plantar Fasciitis: Results of a Confirmatory Randomized Placebo-Controlled Multicenter Study. Am J Sports Med. 2008;36(11):21009. 